\title{
Towards Statistically Representative Atomic Resolution 3D Nano-metrology for Materials Modelling and Catalyst Design.
}

\author{
Lewys Jones ${ }^{1}$, Katherine E. MacArthur ${ }^{1}$, Jolyon Aarons ${ }^{2}$, Chris-Kriton Skylaris ${ }^{2}$, Misbah Sarwar ${ }^{3}$, \\ Dogan Ozkaya ${ }^{3}$ and Peter D. Nellist ${ }^{1}$ \\ 1. Department of Materials, University of Oxford, Oxford UK. \\ 2. School of Chemistry, University of Southampton, Southampton, UK. \\ 3. Johnson Matthey, Reading, UK.
}

Metallic nanoparticles are used widely in a variety of catalyst applications and further improvement in their performance requires materials characterisation at the nano-scale. The aberration corrected scanning transmission electron microscope (STEM) now allows for the routine imaging of metallic nanoparticles at atomic resolution. Especially useful is the annular dark-field (ADF) imaging mode where image contrast is related to sample mass or atomic number. It has been shown that, for pure metals, this approach allows for the number of atoms in atomic-columns to be counted [1] and for the 3D structure of nanoparticles to be revealed [2]. Recently we presented a method for the highthroughput automated analysis of ADF STEM data to produce 3D models of nanoparticles at atomic resolution [3]. By utilising prior knowledge about the structures this allows information such as facet types and surface-atom coordination numbers to be determined on a particle by particle basis (Figure 1) without the need for the multiple frame imaging of tomography. However, in many electron microscopy studies, few unique nanostructures are presented and this may lead to unintended observational bias. The next logical step then is to survey a larger number of particles and to move towards more general measurements of 'average' particle properties.

Here we present our progress towards this goal; the as received commercial catalyst samples were imaged at intermediate magnifications for particle-size analysis ( 1-5Mx), Figure 1. Using this histogram, individual on-axis particles were chosen for high-magnification imaging ( 10-20Mx), atomcounting, and model construction, Figure 2. As this approach yields the total number of atoms per particle these can be directly related back to the sizing histogram. Now we have a collection of 3D atomic models which we believe better represents the overall sample. None of these were so-called 'perfect number' polyhedral and all contain many varied surface atom configurations with a variety of surface nearest-neighbour coordinations and facet types [3].

Next to explore the range of properties of these particles computer modelling is used. First our preliminary atomic model is relaxed at room temperature using Sutton-Chen potential. This relaxation is justified as it accommodates for any mis-measurement in the experimental atom-column positions and any beam-induced heating. During this step we observe little or no structural reconfiguration but often relaxation of bond lengths of a few percent. With the relaxed coordinates, the electronic properties of the particle can be calculated using density-functional theory (DFT). The ONETEP code for large-scale DFT calculations was used to obtain the electronic density and electrostatic potential of the particles (Figure 2).

Using experimentally determined 3D models as inputs for materials modelling allows the inhomogeneous surface electronic properties of catalytic nanoparticles to be explored. Relating the nano-scale surface texture, electronic properties and chemical reactivity of metallic nanoparticle 
catalysts will become key information in designing new and improved systems [4].

References:

[1] J. M. Lebeau and S. Stemmer, Ultramicroscopy 108 (2008), p. 1653-8.

[2] S. Van Aert et at., Nature 470 (2011), p. 374-7.

[3] L. Jones et al., Nano Lett. 14 (2014), p. 6336-41.

[4] The authors acknowledge funding from EU FP7 grant 312483 (ESTEEM2), EPSRC grant

EP/K040375/1 and Johnson Matthey, and S. VanAert and A. DeBacker for their useful discussions.

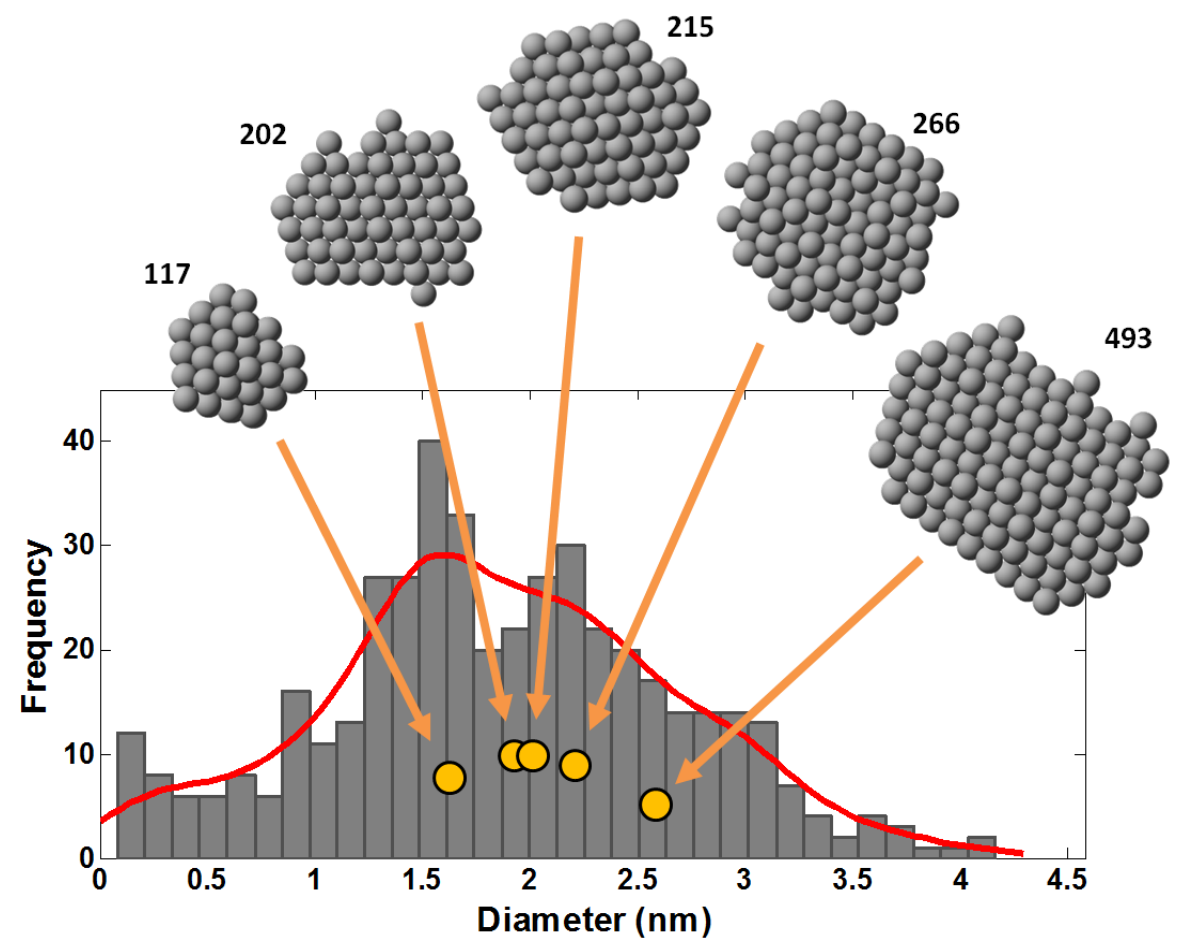

Figure 1. Size-distribution histogram of a commercial batch of $\mathrm{Pt}$ nanoparticles as measured by intermediate magnification ADF STEM (450 particles total).

High-resolution imaging from the same sample was used to rebuild atomic 3D models shown above the histogram. Numbered models indicate the number of atoms in each particle.

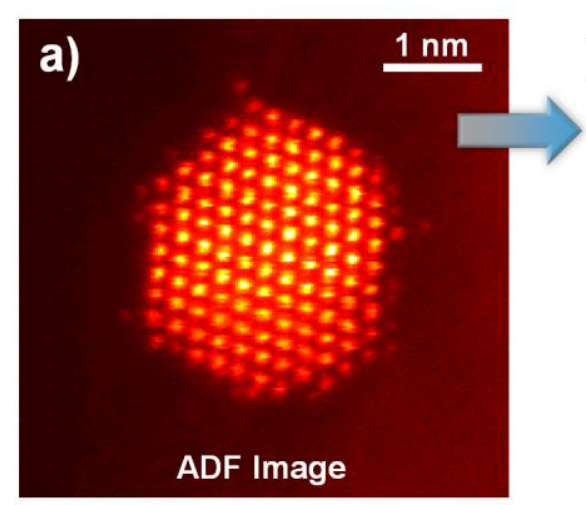

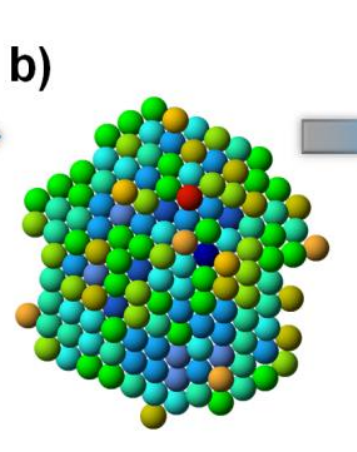

Structural Model
C)

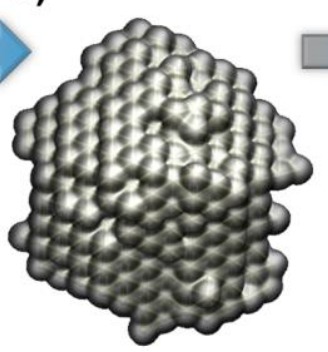

Electronic Density

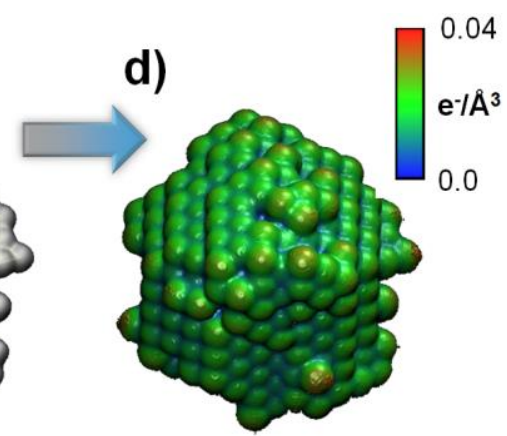

Electrostatic Potential

Figure 2. Workflow of the combined experimental-computational nanoparticle analysis; a) the ADF STEM image recorded with a calibrated detector and used for the column-wise atom-counting, b) the rebuilt structural model from those atom counts (total atoms $=943$, atom colour indicates coordination number [3]), c) the electronic density iso-surface plotted at $0.1 \mathrm{e}^{-} / \AA^{3}$, and d) the surface in c) but coloured using with the values of the electrostatic potential. View d) reveals points of high potential where strong chemical affinity may be expected. 